\title{
Time-resolved fluorescence anisotropy imaging applied to live cells
}

\author{
Klaus Suhling, ${ }^{*}$ Jan Siegel, ${ }^{\dagger}$ Peter M. P. Lanigan, Sandrine Lévêque-Fort, ${ }^{\dagger \dagger}$ Stephen E. D. Webb, ${ }^{*}$ \\ David Phillips, Daniel M. Davis, and Paul M. W. French \\ Departments of Physics, Biological Sciences and Chemistry, Imperial College London, London SW7 2BW, UK
}

Received August 5, 2003

\begin{abstract}
We have developed a wide-field time-resolved imaging system to image quantitatively both the fluorescence lifetime and the rotational correlation time of a fluorophore. Using a polarization-resolved imager, we simultaneously image orthogonal polarization components of the fluorescence emission onto a time-gated intensified CCD. We demonstrate imaging of solvent viscosity variations through the rotational correlation time of fluorescein in a multiwell plate and apply this technique to probe the microviscosity in live cells. @ 2004 Optical Society of America
\end{abstract}

OCIS codes: $170.2520,300.2530,300.6500,000.1430$.

Fluorescence lifetime imaging (FLIM) can report on not only the location of fluorophores but also their biophysical microenvironment. Polarization-resolved fluorescence lifetime measurements of fluorophores excited with linearly polarized light can provide information about their rotational diffusion, which is a function of the viscosity of their local environment and the size of the rotating unit. Recently, frequencydomain FLIM was used to image polarization components sequentially to probe protein interactions by fluorescence resonance energy transfer between identical fluorophores. ${ }^{1}$ We subsequently reported our work extending wide-field picosecond time-gated FLIM to time-resolved fluorescence anisotropy imaging (TR-FAIM) by simultaneously imaging the parallel and perpendicular polarization components of the fluorescence emission. ${ }^{2}$ Acquiring the polarization components simultaneously is faster and reduces artifacts that could be caused by photochemical changes or movement, which may be experienced when the components are acquired sequentially. Here we extend our previous work, which essentially demonstrated qualitative viscosity contrast, by introducing a background subtraction for the calculation of the rotational correlation time and the $G$ factor and by upgrading our experimental system to incorporate a cooled 12-bit digital CCD camera. We can now demonstrate quantitative imaging of solvent viscosity in a multiwell plate and in live cells.

We define the measured time-resolved fluorescence anisotropy $r(t)$ as

$$
r(t)=\frac{I_{\|}(t)-B-G\left[I_{\perp}(t)-B\right]}{I_{\|}(t)-B+2 G\left[I_{\perp}(t)-B\right]},
$$

where $I_{\|}(t)$ and $I_{\perp}(t)$ are the fluorescence intensity decays parallel and perpendicular to the polarization of the exciting light. $G$ accounts for different transmission and detection efficiencies of the imaging system at parallel and perpendicular polarization, ${ }^{2}$ and $B$ is the background discussed below. For a spherical rotor with restricted rotational mobility, $r(t)$ decays monoexponentially and is related to the rotational correla- tion time $\theta$ according to

$$
r(t)=\left(r_{0}-r_{\infty}\right) \exp \left(-\frac{t}{\theta}\right)+r_{\infty},
$$

where $r_{0}$ is the initial anisotropy and $r_{\infty}$ is the limiting anisotropy that accounts for a restricted rotational mobility. For a spherical rotor in an isotropic medium, $\theta$ is directly proportional to the viscosity $\eta$ of the solvent and the volume $V$ of the rotating molecule. ${ }^{2}$

Figure 1 is a schematic of the polarizationresolved imager (Optical Insights) that we use with our wide-field TR-FAIM system. ${ }^{2}$ We applied this system to a multiwell plate sample array of fluorescein in solutions of varying viscosity prepared by mixing an aqueous $\mathrm{NaOH}$ buffer solution and glycerol (fluorescein concentration $1.7 \times 10^{-6} \mathrm{~mol} / \mathrm{dm}^{3}$ ) (Fig. 2). The multiwell plate was placed above a $45^{\circ}$ mirror and imaged with a camera lens [Nikon, $50-\mathrm{mm}$ focal length, numerical aperture (NA) 0.28] in front of the polarization-resolved imager. The samples were excited at $470 \mathrm{~nm}$ with 100 -fs laser pulses, and series of polarization-resolved time-gated fluorescence images were recorded with a cooled 12-bit digital CCD camera (Hamamatsu Orca-ER).

Figure 2(a) shows how the glycerol percentage (by weight) varied from $0 \%$ to $81 \%$. Samples were plated in duplicate. The refractive index of each sample was measured with a refractometer and was

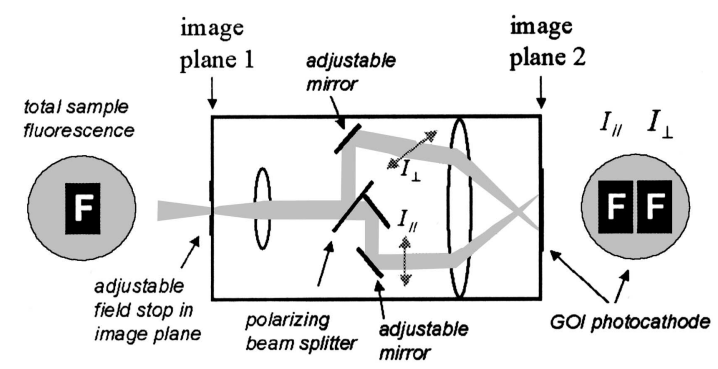

Fig. 1. Schematic of the polarization-resolved imager used to simultaneously acquire orthogonal polarization component images. $^{2}$ 


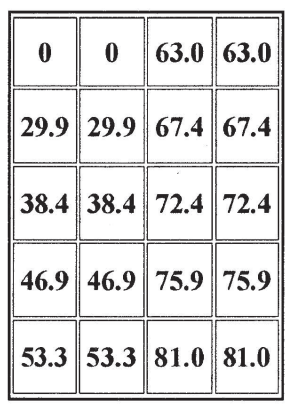

(a)

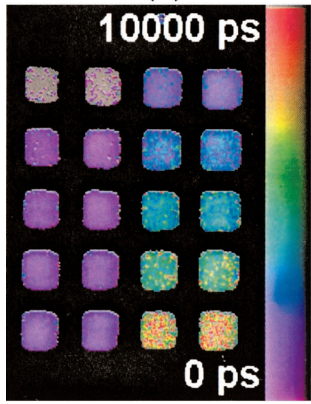

(c)

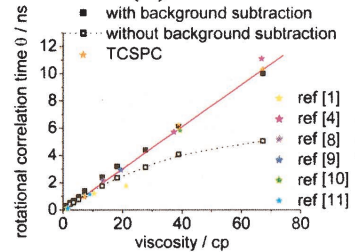

(e)

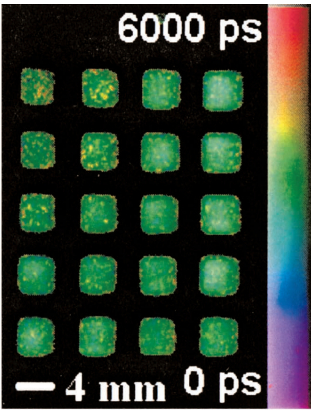

(b)

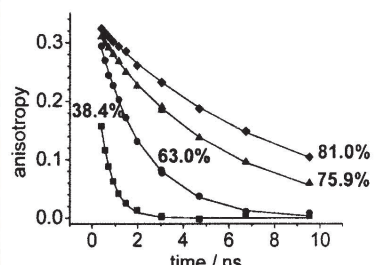

(d)

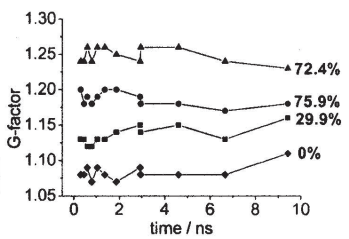

(f)
Fig. 2. (a) Schematic of mixtures of buffer and glycerol (in percent by weight) used to vary the viscosity of the fluorescein environment. (b) Fluorescence lifetime image with the polarization contribution removed. (c) Corresponding rotational correlation time image showing viscosity contrast over more than 1 order of magnitude. (d) Four representative time-resolved anisotropy decays averaged over a well. (e) Plot of the rotational correlation time $\theta$ versus the viscosity $\eta$, including our own time-correlated single-photon counting (TCSPC) data and data points from previous work. ${ }^{1,4,8-11}$ A straight line fit yields a fluorescein radius of $0.54 \mathrm{~nm}$. (f) Plot of the backgroundcorrected $G$ factor of four representative wells as a function of time.

in good agreement with the tabulated value according to its composition. ${ }^{3}$ Figure $2(\mathrm{~b})$ shows the fluorescence lifetime map of this sample array calculated from the decay of the total fluorescence, $I_{\|}(t)+2 G I_{\perp}(t)$. A small fluorescence lifetime decrease (less than 10\%) can be observed as the glycerol content increases, which is consistent with changes in the solvent refractive index. ${ }^{2}$ The fluorescence anisotropy was calculated according to Eq. (1) and fitted to a single exponential [Eq. (2)] with the offset fixed to zero $\left(r_{\infty}=0\right)$ by use of LabVIEW software described previously. ${ }^{2}$ Figure 2(c) shows an image of the rotational correlation time that exhibits clearly discernible contrast over more than 1 order of magnitude. Selected time-resolved fluorescence anisotropy decays (averaged over a well) are shown in Fig. 2(d). The initial anisotropy is close to 0.4, as expected for fluorescein. ${ }^{4}$ We note that the ability to determine rapid depolarization of the fluorescence, such as that observed at low viscosities, is limited by the instrument response time (400 ps FWHM) and the minimum sampling delay after excitation (400 ps). For the nanosecond decay times discussed here, we do not find it necessary to deconvolve the temporal instrument response. ${ }^{2}$ In addition, depolarization by the imaging optics will decrease the measured initial anisotropy. ${ }^{2}$ This effect increases with NA and is not significant for this multiwell plate setup.

Figure 2(e) shows anisotropy values (averaged over a well) as a linear function of viscosity. Assuming a spherical molecule, a hydrodynamic fluorescein radius of $0.54 \mathrm{~nm}$ can be calculated from the gradient $V / k T$ of a straight line fit to the data in Fig. 2(e). This is in good agreement with a hydrodynamic radius of $0.50 \mathrm{~nm}$ derived from a translational diffusion coefficient, obtained from fluorescence recovery after photobleaching measurements, ${ }^{5}$ and a theoretical value of $0.56 \mathrm{~nm}$ for an oblate rotator with $0.7-$ and $0.2-\mathrm{nm}$ axes. $^{6}$

To obtain the excellent quantitative agreement shown in Fig. 2(e), it is necessary to apply background subtraction to the time-resolved data, a correction that was not employed previously. ${ }^{2}$ For a rapidly decaying anisotropy, the effect of this background is small, but for samples with a long rotational correlation time compared with the fluorescence lifetime, the numerator can still be appreciable when the fluorescence has largely decayed. Employing Eq. (1) without a background subtraction $(B=0)$ yields shorter $\theta$ values than expected, and a plot of the rotational correlation time versus the solvent viscosity appears to be nonlinear, as shown by the dotted curve in Fig. 2(e). Using a value $B=1360$, derived from the background of the last time-gated image, however, yields the expected longer rotational correlation times, resulting in a linear plot. ${ }^{7}$ Moreover, data points obtained from previous work ${ }^{1,4,8-11}$ using appropriate viscosity data $^{12,13}$ and our own time-correlated single-photon counting data lie on the line of the backgroundcorrected data points. It is thus essential to use a background subtraction to obtain the correct rotational correlation time.

A $G$-factor image that accounts for different transmission and detection efficiencies at different polarizations for each pixel may be obtained by excitation of the sample with first vertical and then horizontal polarization. ${ }^{2}$ Incorporating background subtraction into the $G$-factor calculation yields

$$
G=\left[\frac{\left(I_{v v}-B\right)\left(I_{h v}-B\right)}{\left(I_{v h}-B\right)\left(I_{h h}-B\right)}\right]^{1 / 2},
$$

where the subscript represents the polarization of the excitation and the emission: $v$ for vertical and $h$ for horizontal. Figure 2(f) presents data from four different wells showing how this background-corrected $G$ factor remains approximately constant over time, as expected. This was not the case in our previous work. ${ }^{2}$

To test this quantitative time-resolved fluorescence anisotropy imaging system with living cells, we imaged 


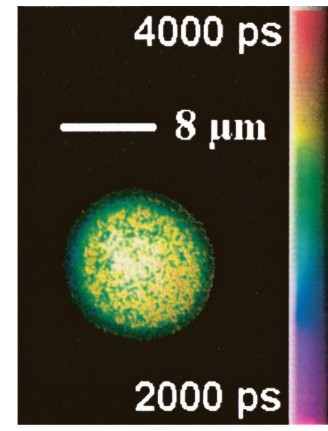

(a)

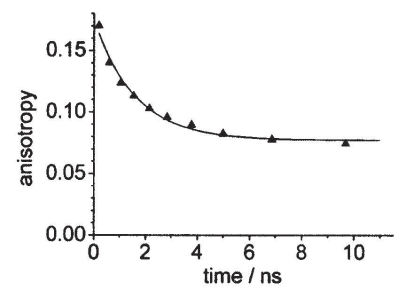

(c)

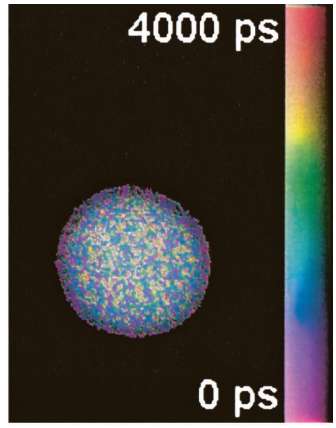

(b)
Fig. 3. (a) FLIM map and (b) rotational correlation time map of CFSE-stained live B cell showing approximately homogeneous distributions throughout the cell. (c) Plot of anisotropy decay (with background subtraction) averaged over the entire cell.

B cells stained with the fluorescein derivative CFSE (carboxyfluorescein diacetate succinimidyl ester, $\mathrm{C}_{29} \mathrm{H}_{19} \mathrm{NO}_{11}, M_{w}=557.5$, Molecular Probes), which can bind to intracellular proteins. The cells were imaged with an inverted wide-field microscope ${ }^{2}$ using a $60 \times 1.2-\mathrm{NA}$ water immersion objective (Olympus UPlanApo). The two-dimensional FLIM map in Fig. 3(a) shows a homogeneous distribution of the CFSE fluorescence lifetime throughout the cell with an average fluorescence lifetime of $3.46 \pm 0.16 \mathrm{~ns}$. The corresponding map of the rotational correlation time is shown in Fig. 3(b), together with the averaged fluorescence anisotropy decay curve in Fig. 3(c). The rotational correlation time averaged over the entire cell is $1.48 \pm 0.15 \mathrm{~ns}$ at $20^{\circ} \mathrm{C}$, which, assuming an effective hydrodynamic radius of $0.62 \mathrm{~nm}$ for CFSE, corresponds to an average cytoplasmic viscosity of $6.0 \mathrm{cp}$. This is approximately what is expected in these conditions: single-point measurements yield $1.2-1.4 \mathrm{cp}$ in Swiss 3T3 fibroblasts at $24^{\circ} \mathrm{C},{ }^{14}$ 2.1-2.5 cp in sea urchin eggs at $22^{\circ} \mathrm{C},{ }^{15}$ and an upper limit of 10-13 cp in Madin-Darby canine kidney cells and Swiss 3T3 fibroblasts. ${ }^{16}$ In addition, we obtained a limiting anisotropy $r_{\infty}=0.08$ that can be interpreted in terms of a hindered rotation of the fluorescent probe as a result of binding in the cytoplasm, a feature that was noted previously. ${ }^{16}$

In conclusion, we have presented quantitative wide-field time-resolved fluorescence anisotropy imag- ing with simultaneous acquisition of the orthogonal polarization components and applied it to imaging solvent viscosity in a multiwell plate and live cell samples. We note that this approach can be extended to overcome the limited depth discrimination in wide-field microscopy by combining it with wide-field optical sectioning techniques such as structured illumination, multibeam multiphoton microscopy, or tandem scanning confocal microscopy.

K. Suhling's e-mail address is k.suhling@imperial. ac.uk.

*Present address, Department of Physics, King's College London, Strand, London WC2R 2LS, UK.

${ }^{\dagger}$ Present address, Instituto de Optica (Consejo Superior de Investigaciones Científicas), C/Serrano 121, Madrid 28006, Spain.

${ }^{+\dagger}$ Present address, Laboratoire de Photophysique Moléculaire, Centre National de la Recherche Scientifique Unité Propre de Recherche 3361, Université Paris Sud Bat 210, Orsay 91405, France.

**Present address, Council for the Central Laboratory of the Research Councils, Daresbury Laboratory, Warrington WA4 4AD, UK.

\section{References}

1. A. H. A. Clayton, Q. S. Hanley, D. J. Arndt-Jovin, V. Subramaniam, and T. M. Jovin, Biophys. J. 83, 1631 (2002).

2. J. Siegel, K. Suhling, S. Lévêque-Fort, S. E. D. Webb, D. M. Davis, D. Phillips, Y. Sabharwal, and P. M. W. French, Rev. Sci. Instrum. 74, 182 (2003).

3. A. A. Newman, Glycerol (Morgan Grampian, London, 1968).

4. J. R. Lakowicz, H. Cherek, B. P. Maliwal, and E. Gratton, Biochemistry 24, 376 (1985).

5. M. B. Mustafa, D. L. Tipton, M. D. Barkley, and P. S. Russo, Macromolecules 26, 370 (1993).

6. G. Porter, P. J. Sadkowski, and C. J. Tredwell, Chem. Phys. Lett. 49, 416 (1977).

7. T. A. Smith, L. M. Bajada, and D. E. Dunstan, Macromolecules 35, 2736 (2002).

8. S. A. Rice and G. A. Kenney-Wallace, Chem. Phys. 47, 161 (1980).

9. A. von Jena and H. E. Lessing, Chem. Phys. 40, 245 (1979).

10. F. V. Bright, C. A. Monnig, and G. M. Hieftje, J. Appl. Phys. 61, 8 (1987).

11. A. S. Verkman, M. Armijo, and K. Fushimi, Biophys. Chem. 40, 117 (1991).

12. J. Jadzyn, G. Czechowski, and T. Stefaniak, J. Chem. Eng. Data 47, 978 (2002).

13. T. Lech, G. Czechowski, and J. Jadzyn, J. Chem. Eng. Data 46, 725 (2001).

14. K. Fushimi and A. S. Verkman, J. Cell Biol. 112, 719 (1991).

15. N. Periasamy, M. Armijo, and A. S. Verkman, Biochemistry 30, 11836 (1991).

16. J. A. Dix and A. S. Verkman, Biophys. J. 57, 231 (1990). 